\title{
The Network Structure of Childhood Psychopathology in International Adoptees
}

\section{Elovainio, Marko}

2018-07

Elovainio , M , Hakulinen , C , Pulkki-Råback , L, Raaska , H \& Lapinleimu , H 2018 , ' The Network Structure of Childhood Psychopathology in International Adoptees ', Journal of Child and Family Studies , vol. 27 , no. 7 , pp. 2161-2170 . https://doi.org/10.1007/s10826-018-1046-z

http://hdl.handle.net/10138/302447

https://doi.org/10.1007/s10826-018-1046-z

publishedVersion

Downloaded from Helda, University of Helsinki institutional repository.

This is an electronic reprint of the original article.

This reprint may differ from the original in pagination and typographic detail.

Please cite the original version. 


\title{
The Network Structure of Childhood Psychopathology in International Adoptees
}

\author{
Marko Elovainio $\mathbb{1}^{1,2} \cdot$ Christian Hakulinen ${ }^{1} \cdot$ Laura Pulkki-Råback $^{3} \cdot$ Hanna Raaska ${ }^{4} \cdot$ Helena Lapinleimu $^{5}$
}

Published online: 28 February 2018

(c) Springer Science+Business Media, LLC, part of Springer Nature 2018

\begin{abstract}
International adoptees are at an increased risk of emotional and behavioral problems, especially those who are adopted at an older age. We took a new approach in our study of the network structure and predictability of emotional and behavioral problems in internationally adopted children in Finland. Our sample was from the on-going adoption study and comprised 778 internationally adopted children (387 boys and 391 girls, mean age 10.5 (SD 3.4) years). Networks were estimated using Gaussian graphical models and lasso regularization for all the children, and separately for those who were adopted at different ages. The results showed that anxiety/depressive symptoms, social problems, and aggressiveness were the most central symptom domains. Somatic symptoms were the least central and had the weakest effect on the other domains. Similarly, aggressiveness, social problems, and attention problems were high in terms of predictability (73-65\%), whereas internalizing problems were relatively low (28-56\%). There were clear but local age-group differences in network structure, symptom centrality, and predictability. According to our findings, network models provide important additional information about the centrality and predictability of specific symptom domains, and thus may facilitate targeted interventions among international adoptees.
\end{abstract}

Keywords Network $\cdot$ Adoption $\cdot \mathrm{CBCL} \cdot$ Behavioral problems

\section{Introduction}

Although most adopted children are well-adjusted (Juffer and van Ijzendoorn 2005), adoption has been identified as an independent risk factor for psychiatric and psychological problems. Studies have reported that several factors in addition to the individual genetic and biosocial background, such as social circumstances prior to adoption, the background of the adoptive family, and relationships between

Marko Elovainio

marko.elovainio@helsinki.fi

1 Department of Psychology and Logopedics, University of Helsinki, Helsinki, Finland

2 National Institute for Health and Welfare, Helsinki, Finland

3 Collegium of Advanced Sciences, University of Helsinki, Helsinki, Finland

4 Department of Child Psychiatry, Helsinki University Central Hospital, Helsinki, Finland

5 Department of Pediatrics and Medicine, Turku University Central Hospital, Turku, Finland the adopted child and his or her family members, may exacerbate developmental difficulties and psychological symptoms (Merz and McCall 2010; O'Connor et al. 2003). It has been shown that a later age at adoption poses a specific mental-health risk (Fensbo 2004), and that children who are younger when they are adopted recover more quickly from the process of adoption and the potential mental-health problems (Beckett et al. 2002). Moreover, girls adopted at more than 12 months of age have more psychological problems than those adopted at a younger age (van Ijzendoorn et al. 2005).

Childhood psychiatric problems have traditionally been classified in terms of externalizing or internalizing behavioral and emotional problems (Achenbach et al. 1987). Internalizing symptoms reflect problems such as anxiety, depression, somatic complaints without a medical cause, and withdrawal from social contact. Externalizing symptoms, on the other hand, manifest in conflicts with other people and their expectations of the children's behavior, and include aggression and rule breaking. The most strongly established method for determining or characterizing behavioral and emotional problems in children is the Child Behavior Checklist questionnaire (CBCL). In terms of 
output, the CBCL gives total scores and separates the internalizing and externalizing symptom scores (secondorder symptom domains), and produces different subscales for "thought", "attention", and "social" problems among 6-18-year-olds (Achenbach and Dumenci 2001).

The common-cause hypothesis, which posits that underlying childhood disorders are dynamic and change depending on the developmental stage, has not been well ascertained (Rutter et al. 2001). The new dynamic-network hypothesis in the field of psychopathology has recently challenged the common-cause approach (Borsboom 2017; Borsboom and Cramer 2013), inspiring a rapidly expanding line of research (Boschloo et al. 2016; Fried et al. 2017). According to this new research stream, psychological symptoms and behavioral problems should be conceptualized as nodes (symptoms or problems) in a network that are mutually dependent via causal associations (edges) (Borsboom and Cramer 2013). Feeling anxious, for instance, could lead to problems with sleeping, which in turn may lead to concentration problems. Hence, psychological symptoms may spread and activate other symptoms in a dynamic network (Boschloo et al. 2015; Epskamp et al. 2012; Frewen et al. 2013; van Borkulo et al. 2014).

The theoretical underpinning in the network perspective is that symptoms are not assumed to be interchangeable, and that their importance, i.e., centrality, in the network should be estimated empirically. Centrality refers to symptoms that have strong connections with many others, the activation of which they are likely to expand through the symptom network once it is activated. Symptoms with fewer connections lie on the periphery of the network and are less important to the entity it describes (Cramer et al. 2010; Cramer et al. 2016; Bringmann et al. 2015; van Borkulo et al. 2015). This research approach has been applied to a range of phenomena in adults, including depression (Boschloo et al. 2016; Fried et al. 2015), anxiety disorders (Beard et al. 2016), psychotic disorders (Isvoranu et al. 2016; Isvoranu et al. 2017), substance abuse (Rhemtulla et al. 2016), the general structure of psychiatric symptomatology (Boschloo et al. 2015), health-related quality of life (Kossakowski et al. 2016), and personality traits (Constantini et al. 2015), but there are significantly fewer studies regarding childhood problems.

All in all, the conceptualization of various psychiatric and psychological symptoms as networks have shed new light on the interconnections of individual symptoms, and thereby on the symptoms that should be the focus of interventions. For instance, symptoms that were closer to the center in the network of depressive symptoms (loss of interest and pleasure, depressed mood, fatigue) were stronger predictors of the onset of a depressive episode than those that were not as central (hypersomnia, decrease in appetite) (van Borkulo et al. 2015a, 2015b).
Although psychiatric symptoms in children have not been examined from a network perspective, a previous study analyzed psychiatric symptomology in adolescents in accordance with the network approach using the Youth selfreport scale on the symptom level (Boschloo et al. 2016). Our study will make a further contribution in testing the network structure at the symptom-domain level in a sample of international adoptees, who have been shown to face an increased risk of psychiatric problems (Elovainio et al. 2015; Raaska et al. 2012). First, we examined the structure of childhood behavioral and emotional problems in terms of its architecture, constructing a symptom-domain network in a sample of international adoptees. Second, given the evidence that older adoptees face an increased psychopathological risk (Raaska et al. 2012), we tested whether the network structures of children who were adopted at an older as opposed to a younger age differed. Third, using the network metrics of centrality (indicating the relative importance of the symptom) and node strength (indicating the strength of its connection with other symptoms) we investigated which items were most significant to behavioral and emotional problems in childhood. Fourth, we evaluated the predictability of the individual symptom domains (how well each symptom in the network is predicted by all the other symptoms). Predictability gives an additional estimate of how relevant a given symptom is, and how it would change after an intervention focused on other symptoms.

\section{Method}

\section{Participants}

This study is part of on-going research covering all children (634 boys and 816 girls, response rate $55.7 \%$ ) (Raaska et al. 2012) adopted internationally through the three legalized adoption organizations in Finland between the years 1985 and 2007. The children were from Asia (China, Thailand, the Philippines, India, Vietnam, Sri Lanka, Pakistan, Nepal, Taiwan, Southern Korea, Israel), Africa (Southern Africa, Ethiopia, Mozambique), America (Columbia, Guatemala, the Dominican Republic, USA, Canada) and Eastern Europe (Russia, Romania, Estonia, Poland).

\section{Procedure}

Data were gathered via self-administered questionnaires both from the adoptive parents and from adoptees aged over 9 years (2007-2009). A subsample of children aged 6-15 years with no missing values in the CBCL symptomdomain measures was used in the current study $(N=758)$. He participants were divided into two age groups (mean ages 1.0 and 4.4 years at the time of the adoption). 
The Ethics Review Committee of the Hospital District of South-West Finland approved this study, and the participating children and their parents gave their written consent for their participation.

\section{Measures}

The Child Behavior Checklist (CBCL) was used to assess behavioral and emotional problems. We grouped the symptoms as internalizing vs. externalizing, each being rated $0=$ "not true", $1=$ "somewhat or sometimes true", or $2=$ "very true or often true". The CBCL has previously been used among internationally adopted children, with good psychometric test results (Dedrick et al. 2008). We used questionnaires compiled for those aged 6-18 and containing 113 questions (Hawk and McCall 2010). From these we constructed the following symptom domains: anxiety/depression, withdrawal/depression, somatic complaints, rule-breaking behavior, social problems, thought problems, attention problems, and aggressive behavior (Achenbach and Rescorla 2001). The Cronbach's alphas of the individual domains were all above 0.97 .

\section{Data Analyses}

The network structure of the CBCL symptom domains was estimated as a Gaussian Graphical Model using the $\mathrm{R}$ qgraph package (Epskamp et al. 2012). The least absolute shrinkage and selection operator (graphical lasso) procedure (Tibshirani 1996) estimates a network in which the nodes are CBCL symptom domains (e.g., aggressiveness, rule breaking, social problems) and the edges are partial correlation coefficients between the nodes. Thus, each edge represents the relationship between two nodes (symptomdomain variables), after controlling for all other relationships in the network. The Graphical lasso minimizes the probability of detecting false positive edges by setting very small edges to zero. The shrinkage parameter is chosen to minimize the extended Bayesian Information Criterion (Chen and Chen 2008), and has been shown accurately to recover underlying network structures (van Borkulo et al. 2014). The graphical representation of networks is based on the Fruchterman-Reingold algorithm that places nodes with stronger and/or more connections closer together. Given that CBCL symptoms are ordered-categorical, the analyses were based on polychoric correlation.

We tested the robustness of the eight-symptom-domain network and of the graph-theoretical measures derived from it using the R-package "bootnet", a bootstrap sampling procedure (Epskamp 2015). We evaluated the stability of the centrality metrics by repeatedly correlating centrality metrics of the original data set with those calculated from subsamples including less and less participants. We then calculated the centrality stability correlation coefficient (CScoefficient) to quantify stability. The CS-coefficient is the maximum number of participants that can be dropped while maintaining $95 \%$ probability that the correlation between centrality metrics from the full data set and the subset data are at least 0.70 . CS-coefficients above 0.50 indicate stable centrality and above 0.25 is recommended for interpreting centrality indices.

We then estimated the centrality of all the symptoms, which represents the connectedness of a given symptom with all the others in the network. The centrality indicators were: (A) node-strength centrality defined as the sum of all associations a given symptom exhibits with all other nodes; (B) betweenness centrality based on the concept that the shortest path length connects any two symptoms (a symptom with high betweenness lies along the shortest path connecting many other symptoms); and (C) closeness centrality as a measure of how close a symptom is to all the other symptoms (Opsahl et al. 2010). The difference in overall connectivity (defined as the weighted sum of the absolute connections (Barrat et al. 2004)) between the networks of those who were adopted at a younger vs. an older age was assessed statistically using the Network Connection Test (NCT), which is implemented in the R-package NCT (Van Borkulo 2015a, 2015b). The NCT is a two-tailed permutation test involving the repeated (100,000 times) calculation of the difference between two groups of randomly regrouped individuals. It results in a distribution under the null hypothesis (on the assumption that both groups are equal), which can be used to test the significance of any difference between the groups. The observed difference is considered significant at the threshold of 0.05 . We assessed the predictability of the individual symptoms using the R-package MGM, which was developed for estimating time-varying mixed graphical models (Haslbeck 2016) version 1.1.-7. The MGMs were estimated via 1regularized (lasso) neighborhood regression, the neighborhood of a node being the set of nodes connected to it.

\section{Results}

Table 1 shows the means and standard deviations of the CBCL symptom domains. The mean age of the participants (girls 50.3\%) was 2.7 years (SD 2.1) at the time of adoption and 10.5 years (SD 3.4 years) at the time of the data collection. The average age at the time of the adoption in the younger group was 1.0 years (SD 0.7) and 4.4 years (SD (0.7) in the older group.

Figures 1a, b show the symptom networks of all the adoptees, and of the two age groups. The edges between the nodes within a network correspond to polychoric partial correlations between the CBCL domains, after controlling 
Table 1 The means and standard deviations of the CBCL symptom domains $(N=784)$

\begin{tabular}{lll}
\hline & Mean & SD \\
\hline Anxiety/depressive symptoms & 2.3 & 3.00 \\
Withdrawal/depressive symptoms & 1.5 & 2.12 \\
Somatic complaints & 1.6 & 2.00 \\
Social problems & 2.5 & 3.06 \\
Thought problems & 1.9 & 2.92 \\
Attention problems & 4.5 & 4.38 \\
Rule-breaking behavior & 2.6 & 4.01 \\
Aggressive behavior & 6.1 & 6.60 \\
$N$ & 784 & \\
\hline
\end{tabular}

for all other domains. The stronger the connection between two nodes, the thicker and more saturated is the edge (the connecting line between two nodes). Positive and negative connections are denoted by green and red edges, respectively. Each node corresponds to a CBCL symptom domain, as shown in Table 1, and is colored according to the secondorder domain to which it is allocated (as shown in Fig. 1a, b). The Fruchterman-Reingold algorithm, which places more strongly connected nodes closer together, is used for node placement in all the networks. The global structure of each one partly reflects the second-order-domain set-up. Domains belonging to the same second-order domain were closely connected and clustered (in predetermined secondorder domains). For instance, aggressive behavior and rule breaking were strongly connected, representing a secondorder domain of externalizing symptoms. Withdrawal and anxiety were also connected, representing a second-order domain of internalizing symptoms, and of the second-orderdomain "other symptoms", attention problems and social problems were connected. Thought problems seemed to be a bridging symptom between internalizing and externalizing.

Our person-dropping stability analysis produced CScoefficients of $0.23,0.62$, and 0.74 for our betweenness, closeness, and strength centrality metrics, respectively. The value for betweenness was below and the others were above the recommended minimum threshold of 0.25 , suggesting that the centrality estimates are somewhat unstable. Differences between those adopted at older as opposed to younger ages seemed to relate to overall connectivity and edge strength. There were fewer but stronger connections between the nodes among those who were adopted at an older age, and the connections more clearly reflect the underlying second-order domain structure.

Figure 2 shows the centrality measures for all the domain networks. The networks of those adopted at an older vs. a younger age differed in centrality on two items: according to all the measures of centrality, the anxiety/depression domain seemed to be the most central among younger but not among the older adoptees, whereas aggressiveness was the most central item among children adopted at an older age. On the other hand, thought problems were less prominent among those who were adopted at a younger age. These results indicate that among all the adoptees, anxiety/ depression, social problems, and aggressiveness may be the symptom domains that affected all the others, and that somatic symptoms had the least effect on the others.

We conducted the Network Comparison Test to examine the differences in overall network structure between the age groups, and could not reject the null hypothesis that the network structure is invariant over subpopulations when the weighted network structures were compared (coefficient $=$ $0.192, p$-value $=0.54)$. These results indicate that the domain-network structure generalizes to different subpopulations quite well. However, it should be noted that this does not rule out the existence of local differences given the limited statistical power to detect them.

Finally, we considered how well the other nodes in the network predicted the individual nodes (symptom domains), meaning that we computed the mean of the conditional distribution of the node at hand with that of its neighbors. Because we were interested in how well a given node could be predicted by all the other nodes in the network, we removed the effects of the intercepts. The results of the predictability analyses are presented in Fig. 3a, b and Table 2. The green edges in Fig. 3a, b indicate positive relationships and the red edges indicate negative relationships. The circle of each color indicates the proportion of explained variance for each node. As expected, there was a close relationship between the parameters of the network model and predictability: if a node was connected to only a few other nodes then the explained variance tended to be low, and the more edges that were connected to a node, the higher the predictability tended to be. Thus, the predictability of aggressiveness was relatively high (0.73), as was that of social problems (0.63) and attention problems (0.65). However, predictability was lower in the case of second-order internalizing symptom domains such as withdrawal (0.41), anxiousness (0.56), and somatic problems (0.28). There were some differences in symptom-domain predictability between those who were adopted at older (Fig. 3b) vs. younger ages. In short, the predictability in domains representing externalizing symptoms (aggressiveness and rule breaking) was stronger among those who were adopted at an older age, whereas internalizing symptoms such as withdrawal and somatic symptoms were more predictable among those adopted at a younger age (Table 2).

\section{Discussion}

We have demonstrated a state-of-the-art approach to modeling the architecture of childhood psychiatric symptomology. 
a

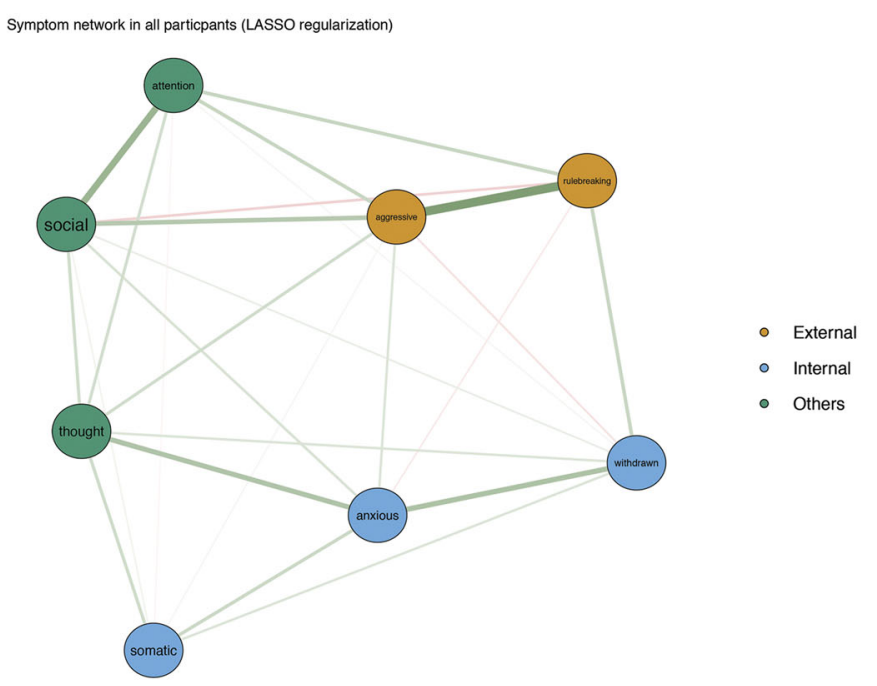

b

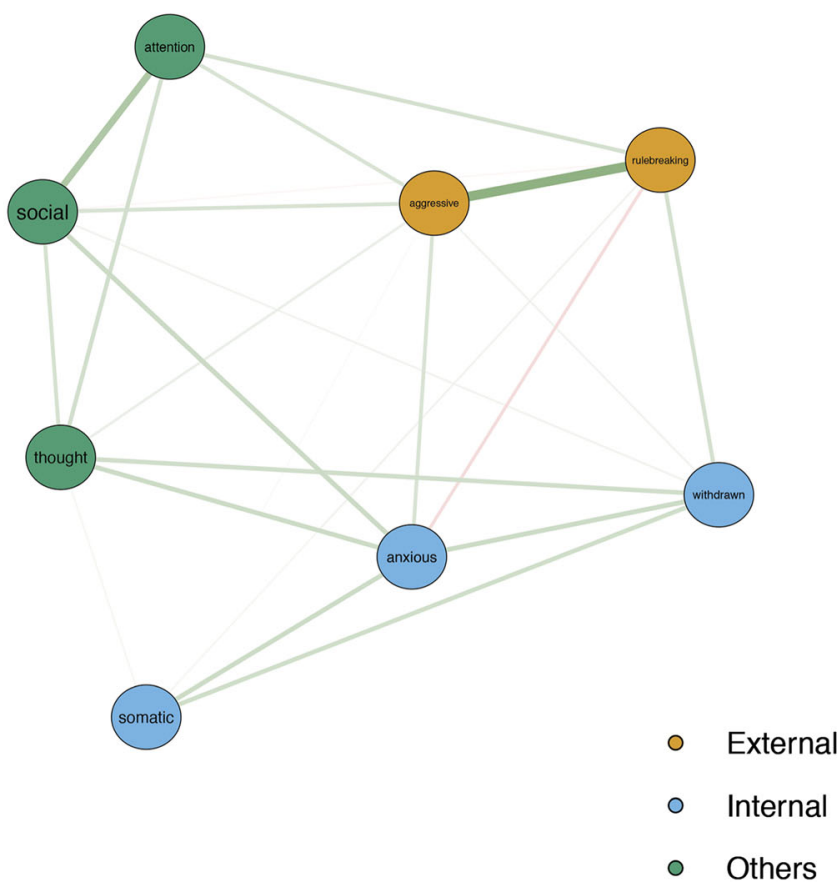

Older children / LASSO regularization

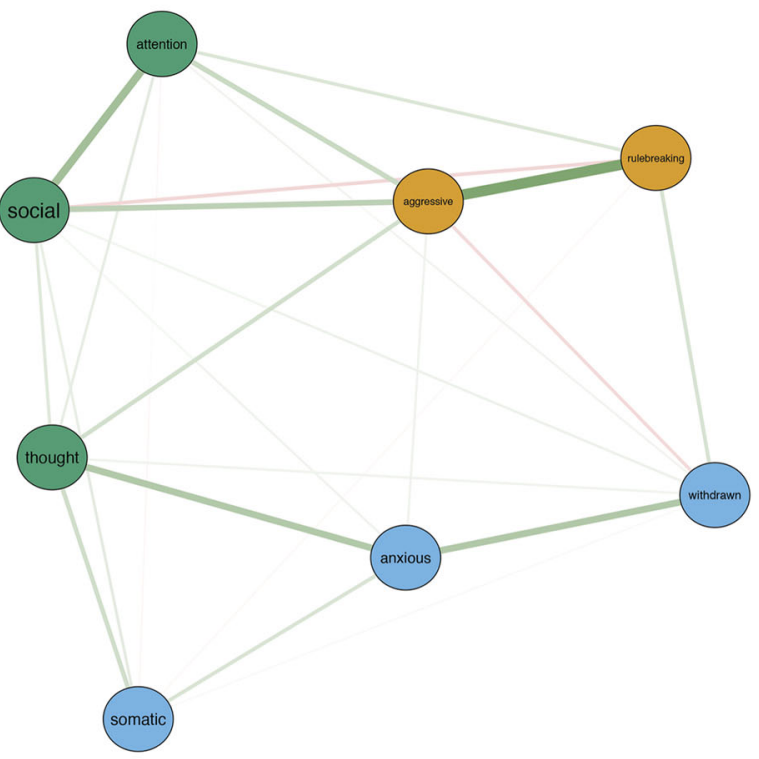

Fig. 1 The network of emotional and behavioral problems in international adoptees as measured by the CBCL in all (a), in those adopted at a younger age and those adopted at an older age (b). The color and

The network structure of international adoptees reflected the predetermined clusters of externalizing and internalizing symptoms relatively well. The connections became more distinct as the child grew older, which is consistent with earlier findings showing that symptoms become more clearly domain-specific at older ages (Dedrick et al. 2008). Externalizing symptoms, such as aggressive behavior, were positioned centrally in the networks according to all the centrality measures, and their predictability was also high. These results thickness of an edge represent the size of the absolute polychoric partial correlation between two nodes. The node colors correspond to the three domains (color figure online)

indicate that symptoms related to the aggressiveness domain potentially affect all the other symptoms and are potentially a good target for interventions. There were local network differences between those adopted at older and younger ages, indicating the centrality of aggressiveness among the former and anxiousness among the latter. According to these results, it would be reasonable to take the age at the time of adoption into account when planning interventions for international adoptees. 


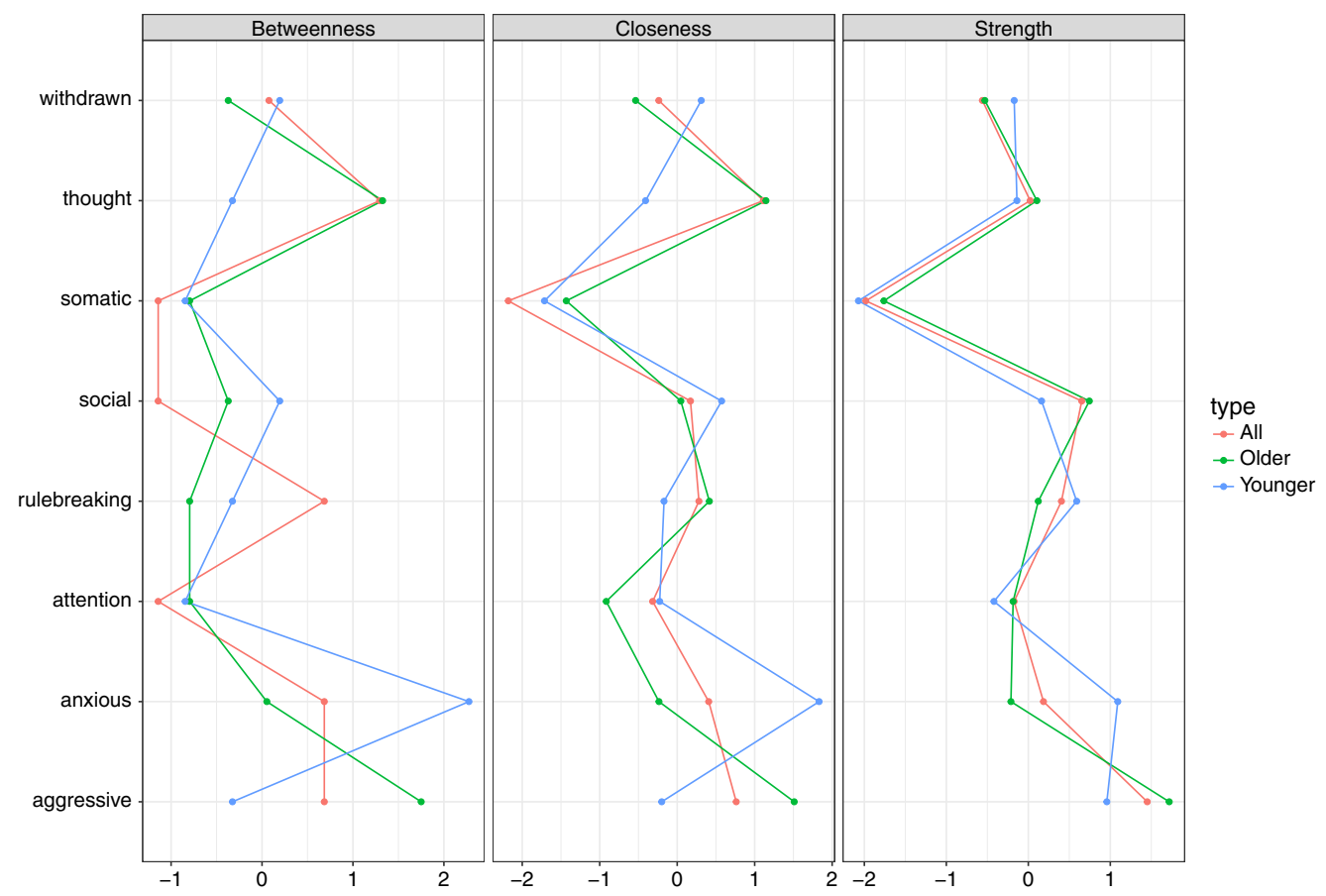

Fig. 2 Centrality measures of symptom domains in all, in those adopted at a younger age and in those adopted at an older age

A number of studies have indicated that internationally adopted adolescents have more mental-health problems than their non-adopted age-mates, adopted adolescents having more externalizing problems in particular (Askeland et al. 2017). It is essential to offer efficient and targeted (treatment) intervention and prevention strategies for this highrisk population already in childhood. Due to increased number of severe attachment problems (disorganized attachment) among adopted children, most of the intervention studies among adopted children have focused on attachment and parental sensitivity. These attachment-based interventions are shown to increase attachment security and well-being of the children (Wright et al. 2015). However, there are findings that severe attachment problems may be linked with concurrent or later behavioral problems and aggression (Zeanah et al. 2016) and it has increased the need to expand the focus of treatment programs also on behavioral problems. Our findings about the centrality of aggressiveness give support on this. In their study using a community sample of adolescents Boschloo and others (Boschloo et al. 2016) showed that externalizing problems had a lot of connections within the same domain and not particular amount connections with other domains. Thus, the centrality of externalizing problems may be typical for international adoptees, but future studies with a case-control design where international adoptees and other children can be efficiently compared are needed.

These results would have been difficult to detect without using the Gaussian Graphical Model (GGM) to determine the network structure for emotional and behavioral problems. Every pairwise interaction is evaluated while controlling for all the other variables, after which the network is regularized by means of lasso penalization. Edges that survive the resulting process are likely to represent real associations. We did not determine the nature or the direction of these associations. Some relationships between symptom domains may reflect potential causal effects, whereas others may be potentially bidirectional. It is highly likely that being aggressive leads to rule-breaking behavior, but not vice versa, whereas aggressive behavior may cause social problems and social problems may also provoke aggressiveness. A similar approach was adopted in a previous study in which the Youth Self Report was used on the item level to test the network structure of emotional and behavioral problems among adolescents (Boschloo et al. 2016). According to the results, the network structure corresponded with the structure that had previously been identified in principal component analyses (symptom domains) (Achenbach and Dumenci 2001). Our findings offer further support for the same theoretical structure in showing that symptom domains within the same second-order domain have more and stronger connections than those in different secondorder domains. However, as in the above-mentioned study (Boschloo et al. 2016), the network-structure analyses also yielded new information about the complex associations between emotional and behavioral problems.

There are pros and cons in testing networks of symptom domains rather than individual symptoms. It is implied in 
a

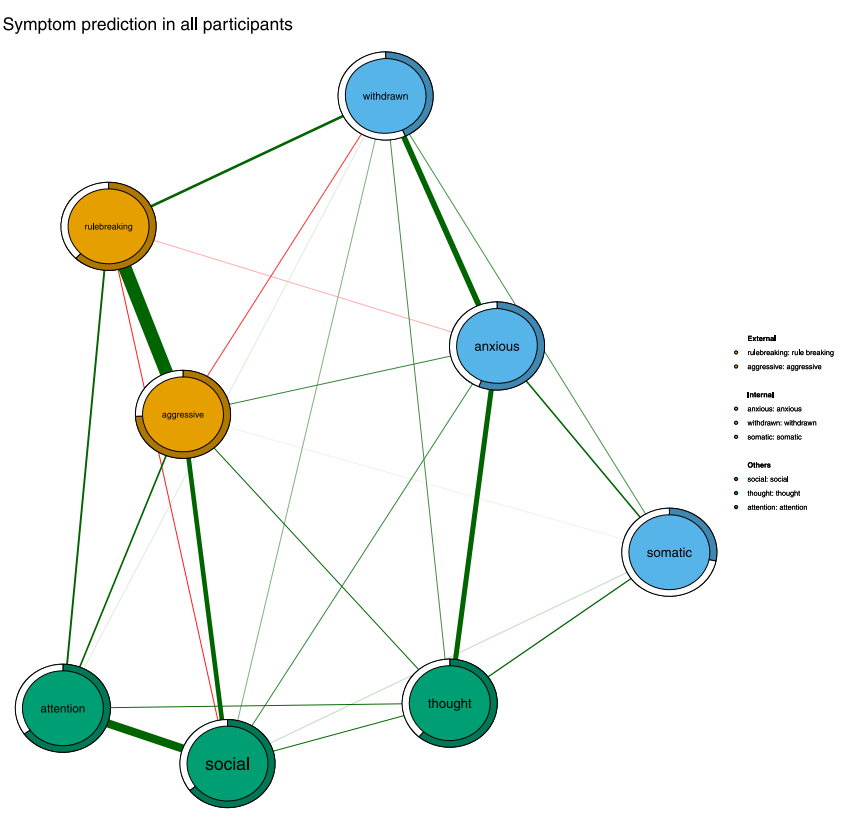

b
Symptom prediction in younger participants

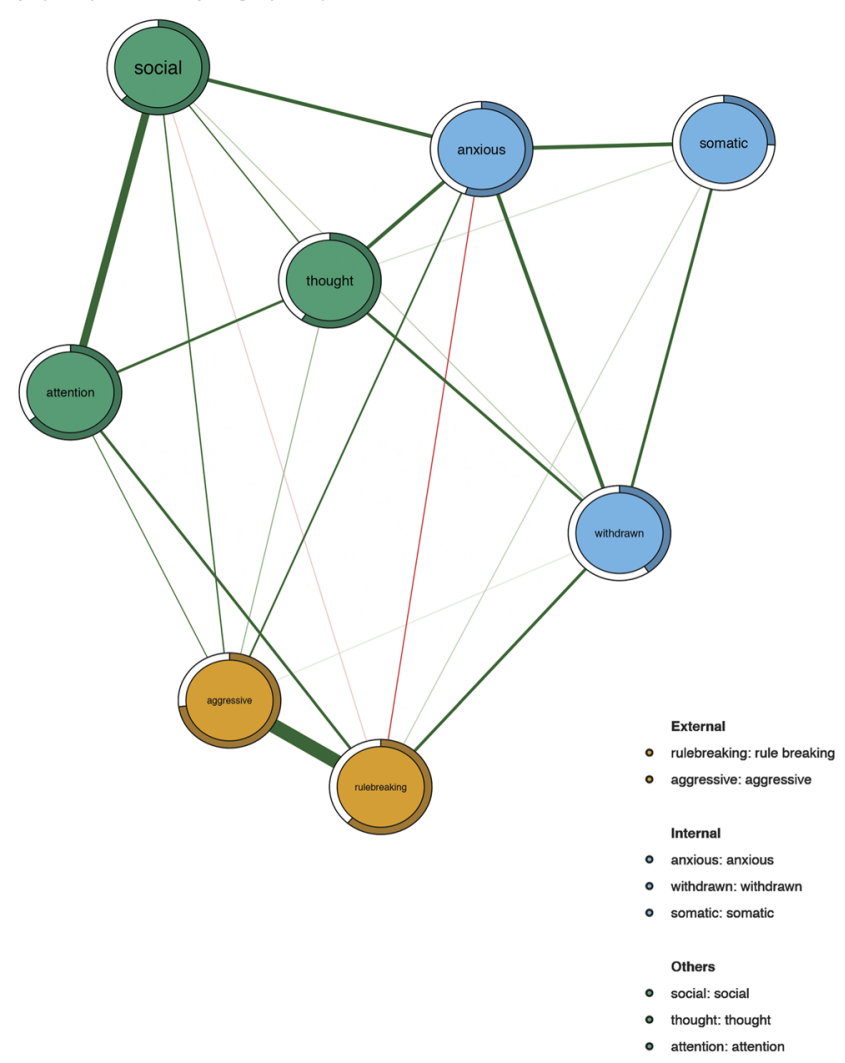

Symptom prediction in older participants

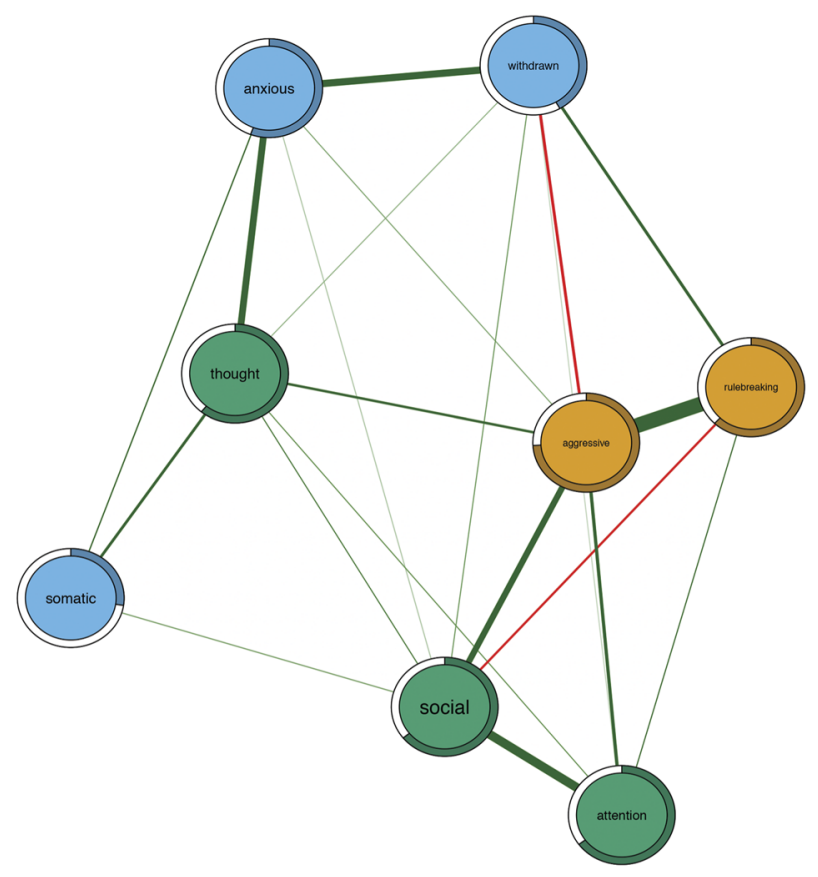

relationships and the red edges negative relationships. The blue ring in all the nodes shows the proportion of explained variance (color figure online)

symptoms that had strong connections with other symptoms in the network more strongly predicted the onset of severe depression than symptoms with few or weak connections.
Fig. 3 The Mixed Graphical Model estimated on the symptom network in all adoptees (a), in those adopted at a younger age and those adopted at an older age (b). The green edges indicate positive

some findings that potentially important information is lost when items are summed to establish scale scores. As Boschloo and others (2016) showed, for instance, depressive 
Table 2 The variance explained of each individual symptom domain in all the adoptees, those who were adopted as older children and those who were adopted as younger children

\begin{tabular}{llll}
\hline Symptom domain & All & $\begin{array}{l}\text { Adopted as } \\
\text { older } \\
(N=778)\end{array}$ & $\begin{array}{l}\text { Adopted as } \\
\text { younger } \\
(N=392)\end{array}$ \\
\hline $\begin{array}{l}\text { Anxiety/depressive } \\
\text { symptoms }\end{array}$ & 0.56 & 0.54 & 0.56 \\
$\begin{array}{l}\text { Withdrawal/depressive } \\
\text { symptoms }\end{array}$ & 0.41 & 0.29 & 0.51 \\
Somatic complaints & 0.28 & 0.26 & 0.32 \\
Social problems & 0.63 & 0.62 & 0.62 \\
Thought problems & 0.53 & 0.58 & 0.48 \\
Attention problems & 0.65 & 0.66 & 0.58 \\
Rule-breaking behavior & 0.58 & 0.59 & 0.48 \\
Aggressive behavior & 0.73 & 0.75 & 0.67 \\
\hline
\end{tabular}

We, therefore, believe that both research and clinical practice could benefit from an approach that specifically focuses on individual problems and their unique role in the network. However, very few scales reflect this thinking, and some individual items measuring individual symptoms are not meant to have any other role than to increase the reliability of the given scale or subscale. Thus, some items may just add noise in the network structure and the analyses may produce artificially complex results. It may nevertheless be reasonable to test the structure on the level of theoretically meaningful measures, in this case on the symptom-domain level. At least these approaches are complementary, as suggested in the above-mentioned study testing the network structure of health-related quality of life (Kossakowski et al. 2016).

In any case, the network approach yields important information about specific associations between symptom domains. One advantage is its capacity to identify the most important nodes in the structure. This type of information may facilitate the formation of hypotheses on the etiological mechanisms underlying the onset and course of psychopathology. Associating symptoms with other symptoms in the network could help in targeting interventions to those children at highest risk. Our centrality analyses revealed that symptoms of aggressiveness and anxiety were the most prominent in all the network structures, particularly among those who were adopted at older and younger ages, respectively. There were no differences between the two groups in overall network connectivity, however. Aggressiveness, problems with social relations, attention problems, rule breaking and anxiety/depression were the symptom domains that the other symptoms in the network predicted most strongly. Factors not included in the network seemed to predict somatic symptoms and withdrawal, especially among those who were adopted at an older age. Thus, it seems that interventions targeted at counteracting aggressiveness should focus on rule-breaking behavior and thought problems. Interventions aimed at reducing anxiety should focus on withdrawn behavior, thought problems and social problems, although there were clear differences between those who were adopted at older as opposed to younger ages.

The network structure in the older group clearly reflected the second-order domain structure, and the predictors probably also belonged to the same second-order domain. Thought problems seemed to be associated with anxiety in both groups. The network structure of those who were adopted at younger age did not reflect the second-order domain structure as clearly, and multiple symptom domains were related. It is thus reasonable to assume that interventions should also focus on multiple or even all symptom domains. A further implication is that the networks of those who were adopted at an older age may be more solid and more difficult to change: according to our findings, most of the symptom domains of those in this group were well explained by the other symptoms domains in the network. Prolonged adverse experiences, or simply getting older, may affect this structuring process, and studies following up international adoptees are needed to assess the differences. Factors that were outside of the network clearly explained more of the symptom domains of those who were adopted at a younger age, hence it would be easier to bring about changes by changing the environment.

\section{Limitations}

A major limitation of this study is the lack of a real prognostic design, which prevented us from drawing any causal conclusions or offering any interpretations related to the temporal order of the associations between the symptom domains. CBCL is an established and widely used measure of childhood psychopathology (Hawk and McCall 2010; Trillingsgaard et al. 2004; Verhulst and Versluis-den Bieman 1995). However, all the information in our study was reported by the parents, and more reliability would have been achieved had we used multiple informants. The CBCL scores we obtained could be considered relatively low compared to the results of previous cross-cultural studies (Viola et al. 2011), especially given that we were focusing on a risk group. Thus, there may be cultural differences in reporting symptoms. Our study focused exclusively on internationally adopted children. Comparisons with non-adopted children in future research could enhance understanding about the pathophysiology of psychiatric symptoms in adoptees. However, even if the levels differ, the associations between the various constructs may not, hence the CBCL may well be a good measure of behavioral problems in Finland and elsewhere.

To conclude, the network structure of emotional and behavioral problems supported the second-order domain structure of the CBCL. Furthermore, the network analyses 
gave potentially important information about the unique role of individual symptom domains and their complex associations, both within and outside the second-order domain. Perceiving emotional and behavioral problems as complex networks may help clinicians to shape their thinking about the various mechanisms and treatment strategies. The differences between the age groups also implies the need for treatment strategies focusing more on those who were adopted at an older age.

Acknowledgements This study was funded by The Foundation for Pediatric Research in Finland, and EVO Grants from Turku University Hospital and the Academy of Finland. We thank the children and families who participated in the study and the Finnish adoption organizations Save the Children Finland and Interpedia, and the city of Helsinki.

Author Contributions M.E.: designed and executed the study, analyzed the data, and wrote the paper. L.P.-R.: collaborated with the design and writing of the study. C.H., H.R., and H.L.: collaborated with the design and writing of the study.

Funding This study was funded by The Foundation for Pediatric Research in Finland, and EVO Grants from Turku University Hospital and the Academy of Finland.

\section{Compliance with Ethical Standards}

Conflict of Interest The authors declare that they have no conflict of interest.

Ethical Approval The Ethics Review Committee of the Hospital District of South-West Finland approved this study.

Informed Consent The participating children and their parents gave their informed written consent for their participation.

\section{References}

Achenbach, T.M., \& Dumenci, L. (2001). Advances in empirically based assessment: revised cross-informant syndromes and new DSM-oriented scales for the CBCL, YSR, and TRF: comment on Lengua, Sadowksi, Friedrich, and Fischer (2001). Journal of Consulting and Clinical Psychology, 69(4), 699-702.

Achenbach, T.M., McConaughy, S.H., \& Howell, C.T. (1987). Child/ adolescent behavioral and emotional problems: Implications of cross-informant correlations for situational specificity. Psychological Bulletin, 101, 213-232.

Achenbach, T.M., \& Rescorla, L.A. (2001). Manual of the ASEBA school_age forms \& profiles. Burlington: University of Vermont, Research Center for Children, Youth \& Families.

Askeland, K.G., Hysing, M., La Greca, A.M., Aaro, L.E., Tell, G.S., \& Sivertsen, B. (2017). Mental Health in Internationally Adopted Adolescents: A Meta-Analysis. Journal of American Academy of Child and Adolescence Psychiatry, 56(3), 203-213. https://doi. org/10.1016/j.jaac.2016.12.009. e201.

Barrat, A., Barthelemy, M., Pastor-Satorras, R., \& Vespignani, A. (2004). The architecture of complex weighted networks. Proceedings National Academy of Science United States A, 101(11), 3747-3752. https://doi.org/10.1073/pnas.0400087101.
Beard, C., Millner, A.J., Forgeard, M.J., Fried, E.I., Hsu, K.J., Treadway, M.T., \& Bjorgvinsson, T. (2016). Network analysis of depression and anxiety symptom relationships in a psychiatric sample. Psychological Medicine, 46(16), 3359-3369. https://doi. org/10.1017/S0033291716002300.

Beckett, C., Bredenkamp, D., Castle, J., Groothues, C., O'Connor, T. G., \& Rutter, M., Romanian Adoptees Study, T. (2002). Behavior patterns associated with institutional deprivation: a study of children adopted from Romania. Journal of Developmental and Behavioral Pediatrics, 23(5), 297-303.

Borsboom, D. (2017). A network theory of mental disorders. World Psychiatry, 16, 5-13.

Borsboom, D., \& Cramer, A.O. (2013). Network analysis: an integrative approach to the structure of psychopathology. Annual Review of Clinical Psychology, 9, 91-121. https://doi.org/10. 1146/annurev-clinpsy-050212-185608.

Boschloo, L., Schoevers, R.A., van Borkulo, C.D., Borsboom, D., \& Oldehinkel, A.J. (2016). The network structure of psychopathology in a community sample of preadolescents. Journal of Abnormal Psychology, 125(4), 599-606. https://doi.org/10. 1037/abn0000150.

Boschloo, L., van Borkulo, C.D., Borsboom, D., \& Schoevers, R.A. (2016). A Prospective study on how symptoms in a network predict the onset of depression. Psychotherapy and Psychosomatics, 85(3), 183-184. https://doi.org/10.1159/000442001.

Boschloo, L., van Borkulo, C.D., Rhemtulla, M., Keyes, K.M., Borsboom, D., \& Schoevers, R.A. (2015). The Network structure of symptoms of the diagnostic and statistical manual of mental disorders. PLoS ONE, 10(9), e0137621 https://doi.org/10.1371/ journal.pone. 0137621 .

Bringmann, L.F., Lemmens, L.H., Huibers, M.J., Borsboom, D., \& Tuerlinckx, F. (2015). Revealing the dynamic network structure of the Beck Depression Inventory-II. Psychological Medicine, 45 (4), 747-757. https://doi.org/10.1017/S0033291714001809.

Chen, J., \& Chen, Z. (2008). Extended Bayesian information criteria for model selection with large model spaces. Biometrika, 95, $759-771$.

Constantini, G., Epskamp, S., Borsboom, D., Perugini, M., Möttus, R., Wldorp, L., \& Cramer, A.O. (2015). State of the aRt personality research: A tutorial on network analysis of personality data in R. Journal of Research in Personality, 54, 13-29.

Cramer, A.O., van Borkulo, C.D., Giltay, E.J., van der Maas, H.L., Kendler, K.S., Scheffer, M., \& Borsboom, D. (2016). Major depression as a complex dynamic system. PLOS ONE, 11(12), e0167490 https://doi.org/10.1371/journal.pone.0167490.

Cramer, A.O., Waldorp, L.J., van der Maas, H.L., \& Borsboom, D. (2010). Comorbidity: a network perspective. Behavioral Brain Sciences, 33(2-3), 137-150. https://doi.org/10.1017/ S0140525X09991567.

Dedrick, R.F., Tan, T.X., \& Marfo, K. (2008). Factor structure of the Child Behavior Checklist/6-18 in a sample of girls adopted from China. Psychological Assessment, 20(1), 70-75. https://doi.org/ 10.1037/1040-3590.20.1.70.

Elovainio, M., Raaska, H., Sinkkonen, J., Makipaa, S., \& Lapinleimu, H. (2015). Associations between attachment-related symptoms and later psychological problems among international adoptees: results from the FinAdo study. Scand Journal of Psychology, 56 (1), 53-61. https://doi.org/10.1111/sjop.12174.

Epskamp, S. (2015). bootnet: Bootstrap methods for various network estimation routines. R-package. Repository CRAN. https://www. r-project.org.

Epskamp, S., Cramer, A.O., Waldorp, L.J., Schmittmann, V., \& Borsboom, D. (2012). qgraph: network visualizations of relationships in psychometric data. Journal of Statistical Softwares, $12,1-18$. 
Fensbo, C. (2004). Mental and behavioural outcome of inter-ethnic adoptees: a review of the literature. European Child and Adolescence Psychiatry, 13(2), 55-63. https://doi.org/10.1007/ s00787-004-0358-2.

Frewen, P.A., Schmittmann, V.D., Bringmann, L.F., \& Borsboom, D. (2013). Perceived causal relations between anxiety, posttraumatic stress and depression: extension to moderation, mediation, and network analysis. European Journal of Psychotraumatology, 4. https://doi.org/10.3402/ejpt.v4i0.20656

Fried, E.I., Bockting, C., Arjadi, R., Borsboom, D., Amshoff, M., Cramer, A.O., \& Stroebe, M. (2015). From loss to loneliness: The relationship between bereavement and depressive symptoms. Journal of Abnormal Psychology, 124(2), 256-265. https://doi. org/10.1037/abn0000028.

Fried, E.I., van Borkulo, C.D., Cramer, A.O., Boschloo, L., Schoevers, R.A., \& Borsboom, D. (2017). Mental disorders as networks of problems: a review of recent insights. Social Psychiatry and Psychiatric Epidemiology, 52(1), 1-10. https://doi.org/10.1007/ s00127-016-1319-z.

Haslbeck, J. (2016). mgm:Estimating Time-Varying MixedGraphical Models. R-package. Repository CRAN. https://www.r-project. org.

Hawk, B., \& McCall, R.B. (2010). CBCL behavior problems of postinstitutionalized international adoptees. Clinical Child and Family Psychology Review, 13(2), 199-211. https://doi.org/10. 1007/s10567-010-0068-x.

Isvoranu, A.M., Borsboom, D., van Os, J., \& Guloksuz, S. (2016). A network approach to environmental impact in psychotic disorder: Brief theoretical framework. Schizophrenia Bulletin, 42(4), 870-873. https://doi.org/10.1093/schbul/sbw049.

Isvoranu, A.M., van Borkulo, C.D., Boyette, L.L., Wigman, J.T., Vinkers, C.H., Borsboom, D., \& Group, I. (2017). A network approach to psychosis: pathways between childhood trauma and psychotic symptoms. Schizophrenia Bulletin, 43(1), 187-196. https://doi.org/10.1093/schbul/sbw055.

Juffer, F., \& van Ijzendoorn, M.H. (2005). Behavior problems and mental health referrals of international adoptees: a meta-analysis. JAMA, 293(20), 2501-2515. https://doi.org/10.1001/jama.293. 20.2501.

Kossakowski, J.J., Epskamp, S., Kieffer, J.M., van Borkulo, C.D., Rhemtulla, M., \& Borsboom, D. (2016). The application of a network approach to Health-Related Quality of Life (HRQoL): introducing a new method for assessing HRQoL in healthy adults and cancer patients. Quality of Life Research, 25(4), 781-792. https://doi.org/10.1007/s11136-015-1127-z.

Merz, E.C., \& McCall, R.B. (2010). Behavior problems in children adopted from psychosocially depriving institutions. Journal of Abnormal Child Psychology, 38(4), 459-470. https://doi.org/10. 1007/s10802-009-9383-4.

O'Connor, T.G., Caspi, A., Defries, J.C., \& Plomin, R. (2003). Genotype-environment interaction in children's adjustment to parental separation. Journal of Child Psychology and Psychiatry, $44(6), 849-856$.

Opsahl, T., Agneessens, F., \& Skvoretz, J. (2010). Node centrality in weighted networks: Generalizing degree and shortest paths. Social Networks, 32, 245-251.

Raaska, H., Elovainio, M., Sinkkonen, J., Matomaki, J., Makipaa, S., \& Lapinleimu, H. (2012). Internationally adopted children in Finland: parental evaluations of symptoms of reactive attachment disorder and learning difficulties - FINADO study. Child Care and Health Development, 38(5), 697-705. https://doi.org/10. 1111/j.1365-2214.2011.01289.x.

Rhemtulla, M., Fried, E.I., Aggen, S.H., Tuerlinckx, F., Kendler, K.S., \& Borsboom, D. (2016). Network analysis of substance abuse and dependence symptoms. Drug and Alcohol Dependence, 161, 230-237. https://doi.org/10.1016/j.drugalcdep.2016.02.005.

Rutter, M., Pickles, A., Murray, R., \& Eaves, L. (2001). Testing hypotheses on specific environmental causal effects on behavior. Psychological Bulletin, 127(3), 291-324.

Tibshirani, R. (1996). Regression shrinkage and selection via the lasso. Journal of Royal Statistical Society, B58, 267-288.

Trillingsgaard, A., Damm, D., Sommer, S., Jepsen, J.R., Ostergaard, O., Frydenberg, M., \& Thomsen, P.H. (2004). Developmental profiles on the basis of the FTF (Five to Fifteen) questionnaireclinical validity and utility of the FTF in a child psychiatric sample. European Child and Adolescence Psychiatry, 13(Suppl 3), 39-63. https://doi.org/10.1007/s00787-004-3006-y.

Van Borkulo, C. (2015). Network comparison test:permutation-based test of differences in strength of networks. https://github.com/ cvborkulo/NetworkComparisonTest.R-package

van Borkulo, C., Borsboom, D., Epskamp, S., Blanken, T.F., Boschloo, L., Schoevers, R.A., \& Waldorp, L.J. (2014). A new method for constructing networks from binary data. Scientific Reports, 4, 5918 https://doi.org/10.1038/srep05918.

van Borkulo, C., Boschloo, L., \& Borsboom, D., et al. (2015a). Association of symptom network structure with the course of depression. JAMA Psychiatry, 72, 1219-1226.

van Borkulo, C., Boschloo, L., Borsboom, D., Penninx, B., Waldorp, L., \& Schoevers, R.A. (2015b). Association of symptom network structure with the course of depression JAMA. Psychiatry, 72 (12), 1219-1224.

van Ijzendoorn, M.H., Juffer, F., \& Poelhuis, C.W. (2005). Adoption and cognitive development: a meta-analytic comparison of adopted and nonadopted children's IQ and school performance. Psychological Bulletin, 131(2), 301-316. https://doi.org/10.1037/ 0033-2909.131.2.301.

Verhulst, F.C., \& Versluis-den Bieman, H.J. (1995). Developmental course of problem behaviors in adolescent adoptees. Journal of American Academy of Child and Adolescence Psychiatry, 34(2), 151-159. doi:S0890-8567(09)63753-9 [pii]10.1097/00004583199502000-00010.

Viola, L., Garrido, G., \& Rescoria, L. (2011). Testing multicultural robusteness of the child behavior checklist in a national epidemiological sample in Uruguai. Journal Abnormal Child Psychology, 39, 897-908.

Wright, B., Barry, M., Hughes, E., Trepel, D., Ali, S., Allgar, V., \& Gilbody, S. (2015). Clinical effectiveness and cost-effectiveness of parenting interventions for children with severe attachment problems: a systematic review and meta-analysis. Health Technology Assessment, 19(52), vii-xxviii. https://doi.org/10.3310/ hta19520. 1-347.

Zeanah, C.H., Chesher, T., \& Boris, N.W. (2016). Practice parameter for the assessment and treatment of children and adolescents with reactive attachment disorder and disinhibited social engagement disorder. Journal of American Academy of Child and Adolescence Psychiatry, 55(11), 990-1003. https://doi.org/10.1016/j.jaa c.2016.08.004. 